\title{
Typ-2-Diabetes
}

\section{Insulin und Inkretin: zwei sich ergänzende Therapieprinzipien}

- Bei der Behandlung von Typ-2-Diabetikern sprechen gewichtige Argumente für eine inkretinbasierte Therapie, aber auch wenn notwendig - für die Hinzunahme von Insulin. Dabei kommt es auf den individuellen Patienten und das Krankheitsstadium an. Ein Nüchternblutzucker von 140 $\mathrm{mg} / \mathrm{dl}$ kann mit einem DPP-4-Hemmer ausreichend gesenkt werden, bei einem Wert von $170 \mathrm{mg} / \mathrm{dl}$ ist dagegen eine Insulingabe indiziert.

Eine gute Einstellung ist möglichst früh anzustreben, um ein negativ formiertes "Glykämisches Gedächtnis" zu vermeiden, betonte Dr. Thorsten Siegmund, München. Als First-line-Medikament hat sich Metformin bewährt. In die zweite Linie sind bereits die neuen inkretinbasierten Präparate vorgerückt.

\section{Vielfältige \\ Kombinationsmöglichkeiten}

Für die Add-on-Gabe eines DPP-4-Hemmers zu Metformin (als Kombipräparat: Velmetia ${ }^{\circledast}$ ) spricht laut Siegmund die einfache Einnahme, gute Verträglichkeit, Gewichtsneutralität sowie die fehlende $\mathrm{Ne}$ benwirkung „Hypoglykämien“.

Lässt sich der $\mathrm{HbA}_{1 \mathrm{c}}$ unter dieser Medikation nicht in den Zielbereich senken, ist die Gabe von Insulin indiziert, betonte Dr. Andreas Lueg, Hameln. Bezüglich der befürchteten Gewichtszunahme verwies er auf die Effekte einer effizienten Diätberatung. In einer Studie konnte durch eine 30-Minuten-Unterrichtung pro Monat das Gewicht unter Insulin konstant gehalten werden (Barrat R et al., 65th ADA 2005: Poster 673).
Siegmund führte für einen DPP-4-Hemmer wie Sitagliptin (z. B. Xelevia ${ }^{\oplus}$ ) an, dass es sogar erwünschte Nebenwirkungen aufweist. Bei KHK wurde mittels des oralen Antidiabetikums die Ejektionsfraktion um rund zehn Prozentpunkte verbessert (Read PA et al., Circ Cardiovasc Imaging 2010; 3: 195-201). Weitere Vorteile von Sitagliptin sind laut Siegmund, dass es gemäß Zulassung mit Insulin kombiniert werden und bei vorhandener Insulinpflichtigkeit dessen Dosis senken kann. Außerdem hat der DPP-4-Hemmer inzwischen die Zulassung bei Typ-2-Diabetikern mit mäßiger bis schwerer Niereninsuffizienz erhalten.

- Reimund Freye

Quelle: Satelliten-Symposium auf der DDGHerbsttagung, Berlin, November 2011 (Veranstalter: Berlin-Chemie)

\section{Rheumatoide Arthritis}

\section{Medikamentenfreie Remissionen sind heute möglich}

- TNFa-Blocker haben den Verlauf bei rheumatoider Arthritis (RA) dramatisch verbessert. Bei einem großen Teil der Betroffenen sind heute Remissionen und ein Aufhalten von Gelenkerosionen möglich.

In Phase-III-Studien ist das Erreichen klinischer Remissionen, definiert als Abfall des Disease Activity Scores (DAS28) $<2,6$, im Verlauf einer Therapie mit TNFaBlockern umfassend dokumentiert. Bei RAPatienten in längerfristiger Remission wird mittlerweile erfolgreich ein Absetzen des

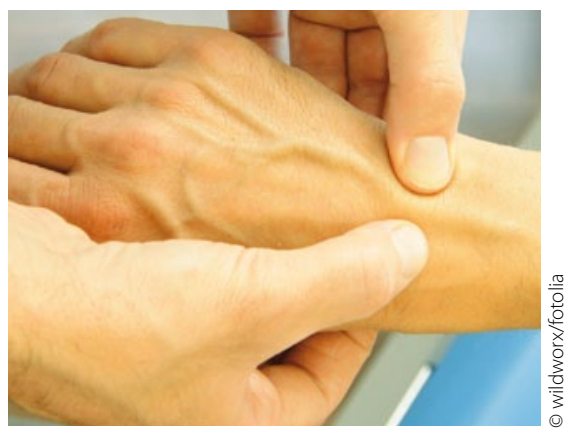

Auch Gelenkerosionen können durch TNFa-Blocker aufgehalten werden.
Biologics oder sogar aller antirheumatischen Medikamente versucht, berichtete Prof. Hanns-Martin Lorenz, Heidelberg. Die besten Daten dazu lägen aus der BeST(Behandel-Strategien-)Studie zu Infliximab (Remicade ${ }^{\circledast}$ ) vor. Die Multicenterstudie verglich bei früher RA vier Therapiestrategien: - sequenzielle Monotherapie,

- stufenweise Kombinationstherapie,

- initiale Kombinationstherapie und

- initiale Kombination unter Einschluss von Infliximab.

\section{Jeder Fünfte in arzneimittelfreier Remission}

Der sofortige Therapiebeginn mit Infliximab erwies sich in mehrfacher Hinsicht als am effektivsten: Die radiologische Progression fiel im 7-jährigen Follow-up am geringsten aus, berichtete Lorenz. Zudem erreichten 45\% der von Anfang an Behandelten eine klinische Remission (DAS44 $<1,6$ ). $17 \%$ konnten Biologic und Methotrexat (MTX) absetzen, ohne dass es zu einem Anstieg des DAS44 kam (Dirven L et al. EULAR
2011; Abstr. SAT0369). Damit gelang bei etwa jedem fünften Patienten eine medikamentenfreie Remission, die im Schnitt 35 Monate anhielt.

Bei Patienten, die nach Absetzen von Infliximab einen Schub erleben, zeigte der TNFa-Blocker meist erneut eine hohe Aktivität. Das belegt eine Post-hoc-Analyse der BeST-Studie nach 7,2-jährigem Follow-up an 104 Patienten, die die Infliximab-Therapie bei einem DAS44 von median 1,3 gestoppt hatten (van den Broek M et al. Ann Rheum Dis 2011). Gut die Hälfte der Patienten (52\%) blieb langfristig in biologicfreier Remission. Bei den Übrigen wurde Infliximab wegen des Anstiegs der Krankheitsaktivität nach median 17 Monaten erfolgreich reinitiiert: $84 \%$ der Patienten sprachen erneut mit einem Abfall des DAS $\leq 2,4$ an.

\footnotetext{
- Katharina Arnheim

Quelle: Symposium „Arzneimittelfreie Remission bei RA und SpA - Vom Wunsch zur Wirklichkeit", 39. Kongress der Deutschen Gesellschaft für Rheumatologie, München, September 2011 (Veranstalter: MSD)
} 